\title{
Production and decay of evolving horizons
}

\author{
Alex B Nielsen ${ }^{1}$ and Matt Visser ${ }^{2}$ \\ ${ }^{1}$ Department of Physics and Astronomy, University of Canterbury, Private Bag 4800, \\ Christchurch, New Zealand \\ ${ }^{2}$ School of Mathematics, Statistics, and Computer Science, Victoria University of Wellington, \\ PO Box 600, Wellington, New Zealand \\ E-mail: alex.nielsen@canterbury.ac.nz and matt.visser@mcs.vuw.ac.nz
}

Received 26 October 2005, in final form 8 May 2006

Published 26 June 2006

Online at stacks.iop.org/CQG/23/4637

\begin{abstract}
We consider a simple physical model for an evolving horizon that is strongly interacting with its environment, exchanging arbitrarily large quantities of matter with its environment in the form of both infalling material and outgoing Hawking radiation. We permit fluxes of both lightlike and timelike particles to cross the horizon, and ask how the horizon grows and shrinks in response to such flows. We place a premium on providing a clear and straightforward exposition with simple formulae. To be able to handle such a highly dynamical situation in a simple manner we make one significant physical restriction-that of spherical symmetry - and two technical mathematical restrictions: (1) we choose to slice the spacetime in such a way that the spacetime foliations (and hence the horizons) are always spherically symmetric. (2) Furthermore, we adopt Painlevé-Gullstrand coordinates (which are well suited to the problem because they are nonsingular at the horizon) in order to simplify the relevant calculations. Of course physics results are ultimately independent of the choice of coordinates, but this particular coordinate system yields a clean physical interpretation of the relevant physics. We find particularly simple forms for surface gravity, and for the first and second law of black hole thermodynamics, in this general evolving horizon situation. Furthermore, we relate our results to Hawking's apparent horizon, Ashtekar and co-worker's isolated and dynamical horizons, and Hayward's trapping horizon. The evolving black hole model discussed here will be of interest, both from an astrophysical viewpoint in terms of discussing growing black holes and from a purely theoretical viewpoint in discussing black hole evaporation via Hawking radiation.
\end{abstract}

PACS numbers: $04.20 .-\mathrm{q}, 04.20 . \mathrm{Cv}$ 


\section{Introduction}

Throughout the 1970s the black hole spacetimes considered in the scientific literature were typically globally stationary and asymptotically flat-with true mathematically precise event horizons (absolute horizons). When accretion was considered it was most often handled in the test-matter limit, where the self-gravitation of the accreting matter was ignored. After the accretion rate was calculated in this approximation one might feed this back into the black hole geometry to allow the spacetime to evolve in a quasi-static manner, but the horizon itself was almost always treated as though the idealized Schwarzschild or Kerr event horizon captured almost all of the relevant physics. The 'membrane paradigm' is perhaps the apex of this quasi-stationary approach [1]. Similarly, calculations concerning Hawking radiation are typically carried out in the test-field limit, where the gravitational field generated by the outgoing Hawking flux is ignored [2]. Once the Hawking flux is calculated, this is set equal to the mass loss rate of the underlying black hole, with the black hole again evolving in a quasi-stationary manner [3].

Those explicit models where exact solutions of the Einstein equations were used to investigate black hole horizons beyond the quasi-stationary approximation generally made rather strong assumptions on the nature of the matter crossing the horizon. For instance, in Oppenheimer-Snyder collapse [4] one is limited to dealing with zero pressure dust, while in the Vaidya solutions [5] one is limited to lightlike radiation crossing the horizon [6] (either incoming radiation, or outgoing radiation, but not both).

More recently, considerable work has been done on relaxing these assumptions in various ways. Motivated partly by advances in numerical relativity, Ashtekar et al [7], and independently Hayward [8], have developed formalisms that address what they variously call 'isolated', 'dynamical' and 'trapping' horizons. These modifications of the notion of event horizon exhibit some but not all of the properties of the 'apparent' horizon defined (for instance) in [9]. (For more technical details, see the recent review [10].) Herein we shall present a particularly simple framework that, while assuming spherical symmetry, does not require asymptotic flatness (or more importantly any notion of stationarity) either of the global spacetime or of the black hole itself. Similarly, no a priori constraints are placed on the nature or quantity of matter crossing the horizon, in either direction. Thus, the framework provides a useful illustration and testing ground for some of the ideas recently popularized by Ashtekar et $a l$ and Hayward.

The choice of Painlevé-Gullstrand coordinates $(\tilde{t}, r, \theta, \phi)$ rather than the more familiar Schwarzschild coordinates $(t, r, \theta, \phi)$ is motivated by a desire to clarify issues such as how the black hole is able to grow despite the supposed 'frozen' nature of Schwarzschild time at the horizon and the related issue of wanting to calculate quantities at the horizon using a coordinate system that does not break down at the horizon. The use of $\tilde{t}$ distinguishes the Painlevé-Gullstrand time coordinate from the Schwarzschild time coordinate $t$. These two coordinates agree at spatial infinity where they correspond to the proper time of observers at infinity. We do not, however, require that such an asymptotically flat region need exist.

We also include some discussion of the relation between our evolving horizons, apparent horizons and the isolated/dynamical/trapping horizons of Ashtekar et al and Hayward. It has recently been conjectured that in a fully quantum gravitational universe true mathematically precise event horizons will not form, only long-lived apparent horizons [11]. This fact has led to claims of a solution to the black hole information paradox [11-14]. While the present work does not directly shed any light on this issue, it does provide a classical arena wherein such ideas can be discussed ${ }^{3}$.

3 For a related quantum framework phrased in terms of the spherically symmetric sub-sector of the coupled Einsteinscalar field system see [15]. 
The organization of the paper is as follows. In section 2, we derive the form of the timedependent, spherically symmetric metric in Painlevé-Gullstrand coordinates and in sections 3 and 4 we use this formulation to derive equations for the rate of change of the horizon radius and area. In sections 6 and 7, we calculate various fluxes and stresses at the horizon using a particular choice of tetrad basis. In sections 5 and 8 , we relate this work to that done by Ashtekar et al and Hayward, and discuss the unified formalism for expanding, stationary and evaporating horizons.

\section{Painlevé-Gullstrand coordinates}

It is well known that any spherically symmetric gravitational field can always be written as

$$
\mathrm{d} s^{2}=g_{t t}(r, t) \mathrm{d} t^{2}+2 g_{r t}(r, t) \mathrm{d} r \mathrm{~d} t+g_{r r}(r, t) \mathrm{d} r^{2}+R(r, t)^{2} \mathrm{~d} \Omega^{2} .
$$

The remaining coordinate freedom in the $r-t$ plane can then be used to reduce the four independent functions above to two. For instance, it is also well known that a static, spherically symmetric gravitational field can always be written, at least locally, in terms of curvature coordinates (also known as Schwarzschild coordinates), as [16, 17]

$$
\mathrm{d} s^{2}=-\mathrm{e}^{-2 \Phi(r)}\left[1-\frac{2 m(r)}{r}\right] \mathrm{d} t^{2}+\frac{\mathrm{d} r^{2}}{1-2 m(r) / r}+r^{2} \mathrm{~d} \Omega^{2},
$$

and furthermore for static spacetimes this representation is quite useful-and in the absence of horizons is even globally useful. Then for a time-dependent spherically symmetric gravitational field it is natural to write

$$
\mathrm{d} s^{2}=-\mathrm{e}^{-2 \Phi(r, t)}\left[1-\frac{2 m(r, t)}{r}\right] \mathrm{d} t^{2}+\frac{\mathrm{d} r^{2}}{1-2 m(r, t) / r}+r^{2} \mathrm{~d} \Omega^{2} .
$$

Indeed, we know that any general spherically symmetric metric can be written in terms of two unknown functions, and here we are just making them time dependent. So, no one can stop you from adopting such a coordinate system, it is just that (as we shall soon argue) this particular coordinate system is less useful than one might at first suppose.

Now for any given slicing of spherically symmetric spacetime into hypersurfaces, and in particular for the choice above, one can look for the existence of apparent horizons in the standard way (see, for instance, [9]). That the apparent horizons depend on the slicing of spacetime was shown most clearly in [18], where non-symmetric slicings of Schwarzschild spacetime were found for which no apparent horizons exist. An explicit example of this can be found in [19]. Because of this particular 'feature' of apparent horizons both Ashtekar et al and Hayward, when discussing general spacetimes, have to introduce considerable technical machinery to avoid pathologies due to potentially ill-behaved slicing of the spacetime.

In contrast, in this paper we will restrict ourselves to spherically symmetric spacetimes and spherically symmetric slicings. So much, though not all, of the technical superstructure simplifies. (The whole point of the present paper is that we want to retain just enough technical machinery to do the job and eliminate as many complications as possible.) Then for the coordinate choice in equation (3) the apparent horizon is informally defined by the implicit relation $2 m(r, t) / r=1$. (We will be more precise and formal below.) That is

$$
2 m\left(r_{H}(t), t\right)=r_{H}(t) .
$$

In the Schwarzschild metric $m(r, t)=M$, where $M$ is a constant, and this just corresponds to the familiar Schwarzschild radius. But once you start asking questions about the evolution $r_{H}(t)$ of this apparent horizon you are plagued with multiple divide by zero errors, as the matrix of metric coefficients, when written in these particular coordinates, is a singular matrix 
at the horizon. The ultimate reason for this behaviour is that Schwarzschild coordinates are reasonably good (or at least not intolerably bad) for probing the geometry of a static black hole, but are not particularly good (in fact, downright awful) for probing the future horizon of an evolving black hole.

To deal with a dynamical black hole, we should use a coordinate system that is well behaved at the apparent horizon. For this paper we will use Painlevé-Gullstrand coordinates which are particularly simple and lead to a particularly nice physical picture. (Other nonsingular coordinate choices such as Eddington-Finkelstein, generalized Vaidya or doublenull coordinates are possible and the physics is entirely equivalent ${ }^{4}$. We concentrate on Painlevé-Gullstrand coordinates because they give a particularly nice form to the equations.)

Starting from (3) we perform the change of coordinates $t \rightarrow \tilde{t}(t, r)$ where $\tilde{t}$ will be the Painlevé-Gullstrand time. Thus,

$$
\mathrm{d} \tilde{t}=\frac{\partial \tilde{t}}{\partial t} \mathrm{~d} t+\frac{\partial \tilde{t}}{\partial r} \mathrm{~d} r \equiv \dot{\tilde{t}} \mathrm{~d} t+\tilde{t}^{\prime} \mathrm{d} r
$$

Substituting in for $\mathrm{d} t$,

$\mathrm{d} s^{2}=-\mathrm{e}^{-2 \Phi(r, t)}\left(1-\frac{2 m(r, t)}{r}\right)\left(\frac{1}{\dot{\tilde{t}}} \mathrm{~d} \tilde{t}-\frac{\tilde{t}^{\prime}}{\dot{\tilde{t}}} \mathrm{~d} r\right)^{2}+\frac{\mathrm{d} r^{2}}{1-2 m(r, t) / r}+r^{2} \mathrm{~d} \Omega^{2}$.

Expanding out and demanding that $g_{r r}=1$ gives the condition

$$
\tilde{t}^{\prime}= \pm \frac{\sqrt{2 m(r, t) / r}}{1-2 m(r, t) / r} \mathrm{e}^{\Phi(r, t)} \dot{\tilde{t}}
$$

That this partial differential equation, which is simply a first-order linear homogeneous equation for $\tilde{t}(t, x)$, always has a unique solution can be shown (for instance) by the method of characteristic curves $[21]^{5}$. Imposing (7) now leads to

$$
g_{\tilde{t} r}= \pm \frac{\mathrm{e}^{-\Phi(r, t)}}{\dot{\tilde{t}}} \sqrt{2 m(r, t) / r}
$$

which can be written as

$$
g_{\tilde{t} r} \equiv \pm c(r, \tilde{t}) \sqrt{2 m(r, \tilde{t}) / r} \equiv v(r, \tilde{t}) .
$$

This implicitly defines $c(r, \tilde{t})$ and leads to

$$
g_{\tilde{t} \tilde{t}}=-\left(c^{2}-v^{2}\right) \text {. }
$$

Thus the final metric, now in Painlevé-Gullstrand form, becomes

$$
\mathrm{d} s^{2}=-c(r, \tilde{t})^{2} \mathrm{~d} \tilde{t}^{2}+[\mathrm{d} r+v(r, \tilde{t}) \mathrm{d} \tilde{t}]^{2}+r^{2} \mathrm{~d} \Omega^{2},
$$

or equivalently

$$
\mathrm{d} s^{2}=-\left[c(r, \tilde{t})^{2}-v(r, \tilde{t})^{2}\right] \mathrm{d} \tilde{t}^{2}+2 v(r, \tilde{t}) \mathrm{d} r \mathrm{~d} \tilde{t}+\mathrm{d} r^{2}+r^{2} \mathrm{~d} \Omega^{2} .
$$

That is, any spherically symmetric spacetime, regardless of whether it is static or not, can always locally be put into this form. Note that, as is usual in Painlevé-Gullstrand coordinates, surfaces of constant $\tilde{t}$ are spatially flat, and there is a natural notion of 'outwards' and 'inwards' associated with increasing or decreasing the $r$ coordinate. Furthermore, four-dimensional asymptotic flatness would correspond to imposing $c \rightarrow 1$ and $v \rightarrow 0$ as $r \rightarrow \infty$. (We will not

4 For an early article presenting an interesting formulation in terms of double-null coordinates see [20].

5 Of course this uniqueness holds only subject to the specific choice $g_{r r}=1$ and a suitable boundary condition (such as $t^{\prime} \rightarrow t$ when $r \rightarrow \infty$ ). What is certainly not unique is the general process of constructing a nonsingular coordinate system at the horizon. Other coordinate systems, such as Eddington-Finkelstein coordinates, are possible and will lead to similar results, that are likely however to differ in small technical details. 
need to impose asymptotic flatness in the discussion that follows.) Also note that the static Schwarzschild case would be given by setting $c=1$ everywhere, while $v(r)=\sqrt{2 M / r}$, with $M$ being a constant. Explicitly, we have

$$
g_{a b}=\left[\begin{array}{c|c}
-\left[c^{2}-v^{2}\right] & v_{j} \\
\hline v_{i} & h_{i j}
\end{array}\right]
$$

and

$$
g^{a b}=\left[\begin{array}{c|c}
-c^{-2} & v^{j} / c^{2} \\
\hline v^{i} / c^{2} & h^{i j}-v^{i} v^{j} / c^{2}
\end{array}\right],
$$

where $h_{i j}$ is the metric of flat Euclidean 3-space in spherical polar coordinates. The ingoing and outgoing radial null curves are defined by $\mathrm{d} s^{2}=0$ and equation (12) gives

$$
\frac{\mathrm{d} r}{\mathrm{~d} \tilde{t}}=-v(r, \tilde{t}) \pm c(r, \tilde{t})
$$

Thus, we can define the location of what we will call the evolving horizon by the very simple and intuitive condition

$$
c(r, \tilde{t})=v(r, \tilde{t})
$$

which has the simple physical interpretation that when $c(r, \tilde{t})<v(r, \tilde{t})$ the outgoing light ray is being dragged backwards to smaller values of $r$. This implicitly defines a function $r_{H}(\tilde{t})$ such that

$$
v\left(r_{H}(\tilde{t}), \tilde{t}\right)=c\left(r_{H}(\tilde{t}), \tilde{t}\right),
$$

which is equivalent to

$$
2 m\left(r_{H}(\tilde{t}), \tilde{t}\right)=r_{H}(\tilde{t}) .
$$

Now the question is: what is the evolution of this function $r_{H}(\tilde{t})$ in terms of the stress-energy at $r_{H}(\tilde{t})$ ?

\section{Surface gravity and the first law}

Using Maple (or some equivalent) to compute the Riemann tensor of the metric (12) in orthonormal components we calculate

$$
R_{\hat{\theta} \hat{\phi} \hat{\theta} \hat{\phi}}=\frac{2 m(r, \tilde{t})}{r^{3}},
$$

so that $m(r, \tilde{t})$ can be physically identified as the Hernandez-Misner mass function [22]. Similarly, since we are dealing with a spherically symmetric spacetime, we could compute the Hawking-Israel quasi-local mass function

$$
m_{\mathrm{HI}}(r, t)=\frac{r}{2}\left[1-g^{a b} \nabla_{a} r \nabla_{b} r\right],
$$

which again leads to the mathematical quantity $m(r, t)$ appearing in the metric being physically identified as the 'mass inside radius $r$ at time $t$ '.

Now since $2 m(r, \tilde{t})=r$ at the evolving horizon, we have

$$
2 \dot{m}\left(r_{H}(\tilde{t}), \tilde{t}\right)+2 m^{\prime}\left(r_{H}(\tilde{t}), \tilde{t}\right) \dot{r}_{H}(\tilde{t})=\dot{r}_{H}(\tilde{t}),
$$

which we can recast as

$$
\dot{m}\left(r_{H}(\tilde{t}), \tilde{t}\right)=\frac{\left[1-2 m^{\prime}\left(r_{H}(\tilde{t}), \tilde{t}\right)\right]}{2} \dot{r}_{H}(\tilde{t}) .
$$


Introducing $\mathcal{A}_{H}=4 \pi r_{H}^{2}$, the area of the evolving horizon, we have

$$
\dot{m}\left(r_{H}(\tilde{t}), \tilde{t}\right)=\frac{1}{8 \pi} \frac{\left[1-2 m^{\prime}\left(r_{H}(\tilde{t}), \tilde{t}\right)\right]}{2 r_{H}(\tilde{t})} \dot{\mathcal{A}}_{H}(\tilde{t}),
$$

where we have seen that the function $m(r)$ can be physically interpreted, due to the spherical symmetry, as the mass contained within a radius $r$. Therefore, this has exactly the form of the first law of black hole mechanics, $\mathrm{d} m=\frac{1}{8 \pi} \kappa \mathrm{d} \mathcal{A}$, though now in a completely dynamical context, provided we agree to focus on partial derivatives with respect to $\tilde{t},{ }^{6}$ and also to identify

$$
\kappa_{H}(\tilde{t})=\frac{\left[1-2 m^{\prime}\left(r_{H}(\tilde{t}), \tilde{t}\right)\right]}{2 r_{H}(\tilde{t})}
$$

as the "surface gravity' ${ }^{7}$. To justify this interpretation of $\kappa_{H}$, it is useful to first define the outward radial null vector

$$
\ell^{a}=\frac{(1, c(r, \tilde{t})-v(r, \tilde{t}), 0,0)}{c(r, \tilde{t})},
$$

and verify that $g_{a b} \ell^{a} \ell^{b}=0$. The overall normalization is chosen to make things simpler below. It is also useful to define the inward radial null vector

$$
n^{a}=\frac{(1,-c(r, \tilde{t})-v(r, \tilde{t}), 0,0)}{c(r, \tilde{t})},
$$

and verify that this is also null $g_{a b} n^{a} n^{b}=0$, and that $g_{a b} \ell^{a} n^{b}=-2$. (This choice of normalization is the most 'symmetric' we have been able to find.)

Because of spherical symmetry we must have

$$
\ell^{b} \nabla_{b} \ell^{a}=\kappa_{\ell} \ell^{a}, \quad n^{b} \nabla_{b} n^{a}=\kappa_{n} n^{a} ;
$$

where the scalars $\kappa_{l}$ and $\kappa_{n}$ are defined everywhere on the spacetime, not just on the evolving horizon. Computing $\kappa_{\ell}$ and $\kappa_{n}$ for the given $\ell^{a}$ and $n^{a}$ yields

$$
\begin{aligned}
& \kappa_{\ell}(r, \tilde{t})=\frac{c^{\prime}(r, \tilde{t})-v^{\prime}(r, \tilde{t})}{c(r, \tilde{t})} \\
& =\frac{1}{2 r} \frac{\left[2 m(r, \tilde{t}) / r-2 m^{\prime}(r, \tilde{t})\right]}{\sqrt{2 m(r, \tilde{t}) / r}}+\frac{c^{\prime}(r, \tilde{t})}{c(r, \tilde{t})}\left[1-\sqrt{\frac{2 m(r, \tilde{t})}{r}}\right] \text {; } \\
& \kappa_{n}(r, \tilde{t})=\frac{-c^{\prime}(r, \tilde{t})-v^{\prime}(r, \tilde{t})}{c(r, \tilde{t})} \\
& =\frac{1}{2 r} \frac{\left[2 m(r, \tilde{t}) / r-2 m^{\prime}(r, \tilde{t})\right]}{\sqrt{2 m(r, \tilde{t}) / r}}+\frac{c^{\prime}(r, \tilde{t})}{c(r, \tilde{t})}\left[1+\sqrt{\frac{2 m(r, \tilde{t})}{r}}\right] .
\end{aligned}
$$

6 If we were to compute the total time derivative of $m\left(r_{H}(\tilde{t}), \tilde{t}\right)$, we would obtain

$$
\frac{\mathrm{d} m\left(r_{H}(\tilde{t}), \tilde{t}\right)}{\mathrm{d} \tilde{t}}=\dot{m}\left(r_{H}(\tilde{t}), \tilde{t}\right)+m^{\prime}\left(r_{H}(\tilde{t}), \tilde{t}\right) \dot{r}_{H}(\tilde{t})=\frac{\dot{r}_{H}(\tilde{t})}{2}
$$

This result, though simple, does not appear to be particularly useful, and does not seem to have a straightforward interpretation in terms of black hole thermodynamics. The best one can apparently do in this regard is to rearrange the above as

$$
\frac{\mathrm{d} m\left(r_{H}(\tilde{t}), \tilde{t}\right)}{\mathrm{d} \tilde{t}}=\frac{\dot{m}\left(r_{H}(\tilde{t}), \tilde{t}\right)}{1-2 m^{\prime}\left(r_{H}(\tilde{t}), \tilde{t}\right)} .
$$

7 For a discussion of situations where the 'entropy = area/4' law might fail see, for instance, [23, 24]. 
On the evolving horizon $\kappa_{\ell}$ reduces to

$$
\kappa_{H}(\tilde{t})=\frac{1-2 m^{\prime}\left(r_{H}(\tilde{t}), \tilde{t}\right)}{2 r_{H}(\tilde{t})},
$$

a formula which has all the appropriate limits and is compatible up to normalization with the results in [17]. In particular, if the geometry is static (time independent) then it is obvious that evolving, apparent, event, isolated, dynamic and trapping horizons coincide, and that $\kappa_{H}$ as defined above reduces to the standard definition. (In [17] the spacetime was in addition assumed asymptotically flat, which we do not need to assume in the current analysis. The asymptotic flatness was then used to motivate a particular non-local normalization for surface gravity. The current definition of 'surface gravity' is in contrast local and only uses information that can be extracted from the vicinity of the evolving horizon itself.)

Now we want to deal with the outermost evolving horizon, which for simplicity we define in the purely intuitive sense. Thus, for purely kinematic reasons

$$
\frac{2 m(r, \tilde{t})}{r}<1 \quad \text { for } \quad r>r_{H}(\tilde{t})
$$

which implies

$$
\left.\left(\frac{2 m}{r}\right)^{\prime}\right|_{H} \leqslant 0 \Rightarrow 1-2 m^{\prime}\left(r_{H}(\tilde{t}), \tilde{t}\right) \geqslant 0
$$

Note that in the case of a spacelike evolving horizon the horizon might, in principle, intersect the constant time slices many times. In such a situation this condition will only identify the outermost intersection. See the appendix on the Vaidya spacetime for more details on this point. Apart from the exceptional case $1-2 m^{\prime}\left(r_{H}(\tilde{t}), \tilde{t}\right)=0$, we see that the surface gravity on the outermost horizon is guaranteed to be positive. Indeed, from the above we can see that the case where $1-2 m^{\prime}\left(r_{H}(\tilde{t}), \tilde{t}\right)=0$ corresponds to $\kappa_{H}(\tilde{t})=0$, the condition for an extremal horizon $^{8}$. For a discussion of the subtleties that can occur once one has multiple nested dynamical horizons, see [25].

Aside. We could also calculate $\kappa_{n}$, corresponding to the ingoing null geodesics, on the evolving horizon. Though there is no difficulty in doing so, there is no clear physical interpretation of the resulting quantity:

$$
\kappa_{n}\left(r_{H}(\tilde{t}), \tilde{t}\right)=\kappa_{H}(\tilde{t})+\frac{2 c^{\prime}\left(r_{H}(\tilde{t}), \tilde{t}\right)}{c\left(r_{H}(\tilde{t}), \tilde{t}\right)} .
$$

Aside. From the defining relations for $\kappa_{\ell}$ and $\kappa_{n}$, the spherical symmetry of the spacetime and the normalization relation between $\ell$ and $n$, it is easy to see that

$$
n^{b} \nabla_{b} \ell^{a}=-\kappa_{n} \ell^{a} ; \quad \ell^{b} \nabla_{b} n^{a}=-\kappa_{\ell} n^{a} ;
$$

or equivalently

$$
\kappa_{n}=\frac{n^{a} n^{b} \nabla_{b} \ell_{a}}{2} ; \quad \kappa_{\ell}=\frac{\ell^{a} \ell^{b} \nabla_{b} n_{a}}{2} .
$$

Aside. From the definition of $\kappa_{\ell}$

$$
\ell^{b} \nabla_{b} \ell^{a}=\kappa_{\ell} \ell^{a}
$$

8 For example, in the case of the Reissner-Nordström spacetime we have $m(r)=M-Q^{2} / 2 r$ and a little bit of algebra is enough to show that the condition $1-2 m^{\prime}\left(r_{H}\right)=0$ is equivalent to the condition $M^{2}=Q^{2}$, the usual condition for an extremal Reissner-Nordström black hole. However, in more general situations the vanishing of the surface gravity is typically taken to be the primary definition of what one means by extremality. 
we see that rescaling the null vector $\ell \rightarrow \alpha \ell$ (which implies $n \rightarrow \alpha^{-1} n$ ) will result in

$$
\kappa_{(\alpha \ell)}=\alpha \kappa_{\ell}+\ell \cdot \nabla \alpha .
$$

So, at the evolving horizon, where $\ell \cdot \nabla \rightarrow c_{H}^{-1} \partial_{t}$, we have

$$
\kappa_{H}(\alpha \ell)=\alpha \kappa_{H}(\ell)+\frac{\dot{\alpha}}{c_{H}} .
$$

That is, among all possible normalizations for $\ell$ (and so implicitly for $n$ ), the one we have chosen is seen to not only be 'symmetric' but additionally to eliminate any explicit time derivatives at the evolving horizon.

\section{Null energy condition}

Now calculate the quantity $G_{a b} \ell^{a} \ell^{b}$. Using Maple (or some equivalent) it is easy to see that

$G_{a b} \ell^{a} \ell^{b}=\frac{2}{c(r, \tilde{t}) r^{2}} \frac{\dot{m}(r, \tilde{t})}{\sqrt{2 m(r, \tilde{t}) / r}}+\frac{2}{c(r, \tilde{t}) r} c^{\prime}(r, \tilde{t})\left(1-\sqrt{\frac{2 m(r, \tilde{t})}{r}}\right)^{2}$,

which we can rearrange to yield

$$
\dot{m}(r, \tilde{t})=\frac{1}{2} c(r, \tilde{t}) r^{2} \sqrt{\frac{2 m(r, \tilde{t})}{r}} G_{a b} \ell^{a} \ell^{b}-c^{\prime}(r, \tilde{t}) \sqrt{2 m(r, \tilde{t}) r}\left(1-\sqrt{\frac{2 m(r, \tilde{t})}{r}}\right)^{2} .
$$

This mass formula applies at any value of $r$. In particular, at the evolving horizon we have the very simple result

$$
\dot{m}\left(r_{H}(\tilde{t}), \tilde{t}\right)=\frac{1}{2} r_{H}^{2}(\tilde{t}) c\left(r_{H}(\tilde{t}), \tilde{t}\right) G_{a b} \ell^{a} \ell^{b} .
$$

Invoking the Einstein equations $G_{a b}=8 \pi T_{a b}$ (with $G_{N} \rightarrow 1$ ), we see

$$
\dot{m}\left(r_{H}(\tilde{t}), \tilde{t}\right)=4 \pi r_{H}^{2}(\tilde{t}) c\left(r_{H}(\tilde{t}), \tilde{t}\right) T_{a b} \ell^{a} \ell^{b} .
$$

Note that this only includes the mass change due to flux across the instantaneous location of the evolving horizon, this does not yet include the effect due to the motion of the evolving horizon.

But in view of equation (22) we have

$$
\dot{r}_{H}(\tilde{t})=\frac{2 \dot{m}\left(r_{H}(\tilde{t}), \tilde{t}\right)}{1-2 m^{\prime}\left(r_{H}(\tilde{t}), \tilde{t}\right)}=\frac{8 \pi r_{H}^{2}(\tilde{t}) c\left(r_{H}(\tilde{t}), \tilde{t}\right) T_{a b} \ell^{a} \ell^{b}}{1-2 m^{\prime}\left(r_{H}(\tilde{t}), \tilde{t}\right)} .
$$

Furthermore, since we have already seen that $1-2 m^{\prime}\left(r_{H}(\tilde{t}), \tilde{t}\right)>0$ for any non-extremal outermost horizon, we deduce that the sign of $\dot{r}_{H}$ is the same as the sign of $T_{a b} \ell^{a} \ell^{b}$. But this is exactly the combination that enters into the null energy condition $\left(T_{a b} \ell^{a} \ell^{b} \geqslant 0\right.$; the NEC) evaluated on the apparent horizon.

That is, as long as the NEC is satisfied the horizon cannot shrink. This is compatible of course with the standard analysis in terms of the Raychaudhuri equation [9], but here we can see the result dropping directly out of one component of the Einstein equations applied to the evolving horizon ${ }^{9}$. We emphasize that the NEC, because it is the weakest of the standard energy conditions, leads to the strongest form of the singularity theorem $[27,28]$. Because

9 Similar violations of the NEC occur at the throats of dynamically evolving wormholes [26]—and one also encounters problems similar to those for evolving horizons if one tries to describe evolving wormholes in Schwarzschild curvature coordinates. Schwarzschild curvature coordinates are ill-adapted to discussing the evolution of a wormhole throat. 
the singularity and area increase theorems can both be phrased in terms of the NEC, we do not really need to consider the WEC, SEC or DEC.

Aside. We can also define the total quasi-local mass inside the evolving horizon by

$$
m_{H}(\tilde{t})=m\left(r_{H}(\tilde{t}), \tilde{t}\right)=r_{H}(\tilde{t}) / 2,
$$

and then

$$
\frac{\mathrm{d} m_{H}}{\mathrm{~d} \tilde{t}}(\tilde{t})=\frac{\dot{m}\left(r_{H}(\tilde{t}), \tilde{t}\right)}{1-2 m^{\prime}\left(r_{H}(\tilde{t}), \tilde{t}\right)}=\frac{4 \pi r_{H}^{2}(\tilde{t}) c\left(r_{H}(\tilde{t}), \tilde{t}\right) T_{a b} \ell^{a} \ell^{b}}{1-2 m^{\prime}\left(r_{H}(\tilde{t}), \tilde{t}\right)} .
$$

This evolution law now characterizes the total mass change, now with contributions both from mass flux across the evolving horizon and from the motion of the evolving horizon itself.

Aside. In contrast, consider the ingoing radial null direction. For $G_{a b} n^{a} n^{b}$, we have the result

$G_{a b} n^{a} n^{b}=\frac{2}{c(r, \tilde{t}) r^{2}} \frac{\dot{m}(r, \tilde{t})}{\sqrt{2 m(r, \tilde{t}) / r}}+\frac{2}{c(r, \tilde{t}) r} c^{\prime}(r, \tilde{t})\left(1+\sqrt{\frac{2 m(r, \tilde{t})}{r}}\right)^{2}$,

which we can rearrange to yield

$\dot{m}(r, \tilde{t})=\frac{1}{2} c(r, \tilde{t}) r^{2} \sqrt{\frac{2 m(r, \tilde{t})}{r}} G_{a b} n^{a} n^{b}-c^{\prime}(r, \tilde{t}) \sqrt{2 m(r, \tilde{t}) r}\left(1+\sqrt{\frac{2 m(r, \tilde{t})}{r}}\right)^{2}$,

but this is nowhere nearly as useful since its form on the evolving horizon is not as nice.

\section{Apparent, isolated, dynamical, trapping and evolving horizons}

While we have used Painlevé-Gullstrand coordinates above to get some very simple and explicit results, we note that similar but coordinate-independent definitions of various types of horizon are given by [7-9]. To see the relation between the evolving horizons at $2 m(r)=r$ and the apparent/isolated/dynamical/trapping horizons of Hawking/Ashtekar/Hayward we need to calculate the expansions $\theta_{\ell}$ and $\theta_{n}$ of the radial null vectors $\ell^{a}$ and $n^{a}$. Defining the expansion of the outgoing and ingoing radial null curves as

$$
\begin{aligned}
& \theta_{\ell}=\left[g^{a b}+\frac{n^{a} \ell^{b}+\ell^{a} n^{b}}{2}\right] \nabla_{a} \ell_{b}=\nabla_{a} \ell^{a}-\kappa_{\ell} ; \\
& \theta_{n}=\left[g^{a b}+\frac{n^{a} \ell^{b}+\ell^{a} n^{b}}{2}\right] \nabla_{a} n_{b}=\nabla_{a} n^{a}-\kappa_{n} ;
\end{aligned}
$$

we get

$$
\begin{aligned}
& \theta_{\ell}=\frac{2(c-v)}{r c}=\frac{2}{r}\left\{1-\sqrt{\frac{2 m}{r}}\right\} \\
& \theta_{n}=\frac{-(c+v)}{r c}=-\frac{2}{r}\left\{1+\sqrt{\frac{2 m}{r}}\right\} .
\end{aligned}
$$

So, we see that $\theta_{\ell}$ changes sign exactly at the evolving horizon $r_{H}$. This is enough to see that the evolving horizons of this paper coincide with the standard definition of apparent horizonindeed all you really need for this to hold is to have a spherically symmetric spacetime with a time slicing that respects the spherical symmetry. 
Now define an 'Ashtekar horizon' $H$ as follows:

(i) $H$ is a three-dimensional, timelike/null/spacelike hypersurface.

(ii) On $H$ the expansion of $n^{a}$ is negative, $\theta_{n}<0$.

(iii) On $H$ the expansion of $\ell^{a}$ is zero, $\theta_{\ell}=0$.

The null case represents an 'isolated horizon' and the spacelike case represents a dynamical horizon. (This is the astrophysically relevant case where accretion dominates over Hawking evaporation.) The timelike case corresponds to a situation where Hawking evaporation dominates over accretion - this might be referred to as an 'evaporating horizon'. From the explicit formulae for the expansions $\theta_{\ell}$ and $\theta_{n}$ it is clear that our evolving horizon is also a horizon in the sense of Ashtekar et al.

Finally, a 'Hayward horizon', or more precisely a future outer trapping horizon, replaces condition $i$ with

$$
\mathcal{L}_{n} \theta_{\ell}<0 \text {. }
$$

But explicitly

$$
\mathcal{L}_{n} \theta_{\ell}=(n \cdot \nabla)\left[\frac{2}{r}\left\{1-\sqrt{\frac{2 m}{r}}\right\}\right],
$$

and so on the evolving horizon

$$
\left.\mathcal{L}_{n} \theta_{\ell}\right|_{H}=-\frac{2}{r_{H}}(n \cdot \nabla)\left[\sqrt{\frac{2 m}{r}}\right]_{H}=-\frac{1}{r_{H}}(n \cdot \nabla)\left[\frac{2 m}{r}\right]_{H} .
$$

On the evolving horizon we also have

$$
(n \cdot \nabla) \rightarrow\left(c_{H}^{-1} \partial_{t}-2 \partial_{r}\right),
$$

whence

$$
\left.\mathcal{L}_{n} \theta_{\ell}\right|_{H}=-\frac{1}{r_{H}}\left[\frac{2 \dot{m}}{c r}-2\left(\frac{2 m}{r}\right)^{\prime}\right]_{H} .
$$

That is

$$
\left.\mathcal{L}_{n} \theta_{\ell}\right|_{H}=-\frac{1}{r_{H}^{2}}\left[\frac{2 \dot{m}}{c}+2\left(1-2 m^{\prime}\right)\right]_{H} .
$$

But we have already seen, on purely kinematic grounds, how to relate $\left.\dot{m}\right|_{H}$ to $\dot{r}_{H}$, so

$$
\left.\mathcal{L}_{n} \theta_{\ell}\right|_{H}=-\frac{1-2 m_{H}^{\prime}}{r_{H}^{2}}\left[2+\frac{\dot{r}_{H}}{c_{H}}\right] .
$$

We have furthermore already argued that $1-\left.2 m^{\prime}\right|_{H}>0$, so under the rather mild condition that $\dot{r}_{H}>-2 c_{H}$ we have $\left.\mathcal{L}_{n} \theta_{\ell}\right|_{H}<0$, in which case our evolving horizon is also a Hayward-style future outer trapping horizon.

In the situation where $\dot{r}_{H}<-2 c_{H}$ we have a somewhat unusual type of spacelike evolving horizon - in the present context this would correspond to rapid 'evaporation' of the horizon. Since the evolution of the horizon now lies outside the lightcone, evolving forward along curves of $n^{a}$ does not take us from a region of positive $\theta_{\ell}$ to negative $\theta_{\ell}$. Timelike observers are still instantaneously forced to move inwards, but they immediately escape the horizon-so that subsequently some of them may turn around and escape to 'infinity'. Indeed, once the observer exits they will not be able to re-enter the horizon unless the rate of evaporation slows down.

In short, the very simple and intuitively clear definition of evolving horizon that we advocate in this paper is compatible with all the standard definitions (apparent, isolated, dynamic, evaporating, trapping) common in the literature. The one thing the evolving horizon is not is that it is definitely not an event horizon (absolute horizon). And one very important 
message to take from this entire discussion is that event horizons (in the strict technical sense) are not essential to investigating black hole physics [11,29].

\section{Energy-momentum in a natural tetrad basis}

To understand the energy-momentum fluxes and stresses at the evolving horizon it is extremely useful to adopt a suitable orthonormal tetrad basis. Once you have picked a tetrad, which is effectively a choice of privileged observer, you can begin to ask questions about densities, fluxes and stresses measured by that observer.

To construct an appropriate 'natural' tetrad, start with the observation that the radial null curves are given by

$$
\frac{\mathrm{d} r}{\mathrm{~d} t}=-v \pm c
$$

which implies that the vector $(1,-v, 0,0)^{a}$ is certainly timelike. In fact,

$$
V^{a}=\frac{(1,-v, 0,0)}{c}=\frac{\ell^{a}+n^{a}}{2}
$$

is easily seen to be a timelike unit vector. (In an analogue interpretation of the metric, $V$ would be the 4-velocity of the 'medium', and our natural observers would be comoving with the medium [30].) Similarly,

$$
S^{a}=(0,1,0,0) ; \quad S_{a}=(v, 1,0,0)
$$

are easily seen to be a spacelike unit vector that is orthogonal to $V$. We take these as the first two elements of the tetrad. Specifically, we set

$$
e_{\hat{a}}^{a}=\left(V^{a}, S^{a}, \hat{\theta}^{a}, \hat{\phi}^{a}\right) .
$$

Note that

$$
\begin{aligned}
& V^{a}+S^{a}=\frac{(1, c-v, 0,0)}{c}=\ell^{a} ; \\
& V^{a}-S^{a}=\frac{(1,-c-v, 0,0)}{c}=n^{a},
\end{aligned}
$$

where $\ell$ is the outgoing null vector we previously chose to make the surface gravity computation simple. One may easily compute

$$
\begin{array}{ll}
(V \cdot \nabla) V=\frac{c^{\prime}}{c} S ; & (V \cdot \nabla) S=\frac{c^{\prime}}{c} V ; \\
(S \cdot \nabla) V=-\frac{v^{\prime}}{c} V ; & (S \cdot \nabla S)=-\frac{v^{\prime}}{c} V .
\end{array}
$$

Thus, an observer who 'moves with the medium' is not in geodesic motion except in the special case $c^{\prime}=0$.

Writing $\hat{t}^{a}=V^{a}$ and $\hat{r}^{a}=S^{a}$ and using this natural tetrad (and its inverse as determined by Maple ${ }^{10}$ ), the orthonormal components of the Einstein tensor (at any point in the spacetime)

${ }^{10}$ We particularly wish to warn readers against over enthusiastic use of the frame command in Maple or its equivalent in other packages. The frame function will provide some orthonormal tetrad for the specified metric, but the orthonormal tetrad it provides is certainly not unique, and may not always be the most useful. In particular, if the spacetime contains any form of horizon, the vectors in the orthonormal tetrad provided by the frame command quite often have divergent components at the horizon. The fact that the tetrad is not unique is of course an unavoidable consequence of local Lorentz invariance, and the divergence of the components of the tetrad is a side effect of attempting a ' $v=c^{\prime}$ Lorentz transformation at the horizon, so similar problems will show up in any symbolic computation package. We have found it best to determine a suitable well-behaved tetrad 'by hand' and then explicitly feed it to the symbolic manipulation program for further computations. 
are easily calculated to be

$$
\begin{aligned}
G_{\hat{t} \hat{t}} & =\frac{2 m^{\prime}}{r^{2}}+\frac{2 c^{\prime}}{c r} \frac{2 m}{r} \\
G_{\hat{t} \hat{r}} & =-\frac{2 c^{\prime}}{c r} \sqrt{\frac{2 m}{r}} \\
G_{\hat{r} \hat{r}} & =-\frac{2 m^{\prime}}{r^{2}}+\frac{2 c^{\prime}}{c r}+\frac{2 \dot{m}}{c r^{2}} \frac{1}{\sqrt{2 m / r}} .
\end{aligned}
$$

The only 'complicated' component of the Einstein tensor is the transverse one:

$$
\begin{aligned}
G_{\hat{\theta} \hat{\theta}}=G_{\hat{\phi} \hat{\phi}}= & -\frac{m^{\prime \prime}}{r}+\frac{c^{\prime \prime}}{c}\left(1-\frac{2 m}{r}\right)-\frac{3 c^{\prime} m^{\prime}}{c r}+\frac{c^{\prime}}{c r}\left(1+\frac{m}{r}\right) \\
& +\sqrt{\frac{2 m}{r}}\left\{\frac{\dot{m}}{4 m c r}\left[1+\frac{2 c^{\prime} r}{c}-\frac{r m^{\prime}}{m}\right]+\frac{1}{2 c}\left[\frac{\dot{m}^{\prime}}{m}+\frac{2 \dot{c}^{\prime}}{c}-\frac{2 \dot{c} c^{\prime}}{c^{2}}\right]\right\} .
\end{aligned}
$$

The Einstein tensor at the evolving horizon simplifies rather drastically:

$$
\begin{aligned}
\left.G_{\hat{t} \hat{t}}\right|_{H} & =\frac{2 m^{\prime}}{r^{2}}+\frac{2 c^{\prime}}{c r} ; \\
\left.G_{\hat{\imath} \hat{r}}\right|_{H} & =-\frac{2 c^{\prime}}{c r} ; \\
\left.G_{\hat{r} \hat{r}}\right|_{H} & =-\frac{2 m^{\prime}}{r^{2}}+\frac{2 c^{\prime}}{c r}+\frac{2 \dot{m}}{c r^{2}} ;
\end{aligned}
$$

with the only 'complicated' component being

$\left.G_{\hat{\theta} \hat{\theta}}\right|_{H}=\left.G_{\hat{\phi} \hat{\phi}}\right|_{H}=-\frac{m^{\prime \prime}}{r}-\frac{3 c^{\prime} m^{\prime}}{c r}+\frac{3 c^{\prime}}{2 c r}+\frac{\dot{m}}{2 c r^{2}}\left[1+\frac{2 c^{\prime} r}{c}-2 m^{\prime}\right]+\frac{1}{c}\left[\frac{\dot{m}^{\prime}}{r}+\frac{\dot{c}^{\prime}}{c}-\frac{\dot{c} c^{\prime}}{c^{2}}\right]$.

Now write the Einstein equations as $G_{a b}=8 \pi G_{N} T_{a b}$ and adopt units where $G_{N}=1$. Then (at the evolving horizon),

$$
\begin{aligned}
& \rho_{H}=T_{\hat{t} \hat{t}}=\frac{G_{\hat{t} \hat{t}}}{8 \pi}=\frac{m^{\prime}}{4 \pi r^{2}}+\frac{c^{\prime}}{4 \pi r c} ; \\
& f_{H}=T_{\hat{\imath} \hat{r}}=\frac{G_{\hat{\imath} \hat{r}}}{8 \pi}=-\frac{c^{\prime}}{4 \pi r c} ; \\
& p_{r, H}=T_{\hat{r} \hat{r}}=\frac{G_{\hat{r} \hat{r}}}{8 \pi}=-\frac{m^{\prime}}{4 \pi r^{2}}+\frac{c^{\prime}}{4 \pi r c}+\frac{\dot{m}}{4 \pi r^{2} c} ;
\end{aligned}
$$

with again the only real complication being

$$
\begin{aligned}
p_{t, H}=T_{\hat{\theta} \hat{\theta}}= & T_{\hat{\phi} \hat{\phi}}=\frac{G_{\hat{\theta} \hat{\theta}}}{8 \pi}=-\frac{m^{\prime \prime}}{8 \pi r}-\frac{3 c^{\prime} m^{\prime}}{8 \pi c r}+\frac{3 c^{\prime}}{16 \pi c r} \\
& +\frac{\dot{m}}{16 \pi c r^{2}}\left[1+\frac{2 c^{\prime} r}{c}-2 m^{\prime}\right]+\frac{1}{8 \pi c}\left[\frac{\dot{m}^{\prime}}{r}+\frac{\dot{c}^{\prime}}{c}-\frac{\dot{c} c^{\prime}}{c^{2}}\right] .
\end{aligned}
$$

Note that these must be interpreted as the density, flux and pressures as measured by the particular observer moving with 4-velocity $V^{a}$. In terms of these quantities we have the simple results

$$
\dot{m}=4 \pi r^{2} c\left(\rho_{H}+p_{r, H}+2 f_{H}\right)=4 \pi r^{2} c\left[T_{a b} \ell^{a} \ell^{b}\right]_{H},
$$


and

$$
m^{\prime}=4 \pi r^{2} c\left(\rho_{H}+f_{H}\right)=4 \pi r^{2} c\left[T_{a b} \ell^{a} V^{b}\right]_{H} .
$$

This now implies that the evolving horizon shifts according to the formula

$$
\dot{r}_{H}=\frac{8 \pi c_{H} r_{H}^{2}\left(\rho_{H}+p_{r, H}+2 f_{H}\right)}{1-8 \pi r_{H}^{2}\left(\rho_{H}+f_{H}\right)}=\frac{8 \pi c_{H} r_{H}^{2}\left[T_{a b} \ell^{a} \ell^{b}\right]_{H}}{1-8 \pi r_{H}^{2}\left[T_{a b} V^{a} \ell^{b}\right]_{H}} ;
$$

and similarly

$$
\dot{m}_{H}=\frac{4 \pi c_{H} r_{H}^{2}\left(\rho_{H}+p_{r, H}+2 f_{H}\right)}{1-8 \pi r_{H}^{2}\left(\rho_{H}+f_{H}\right)}=\frac{4 \pi c_{H} r_{H}^{2}\left[T_{a b} \ell^{a} \ell^{b}\right]_{H}}{1-8 \pi r_{H}^{2}\left[T_{a b} V^{a} \ell^{b}\right]_{H}} .
$$

Furthermore, the surface gravity is

$$
\kappa_{H}=\frac{1-2 m^{\prime}}{2 r_{H}}=\frac{1-8 \pi\left(\rho_{H}+f_{H}\right)}{2 r_{H}}=\frac{1-8 \pi\left[T_{a b} V^{a} \ell^{b}\right]_{H}}{2 r_{H}} .
$$

This now provides explicit formulae for the evolution and properties of the horizon in terms of various quantities (densities, fluxes, pressures, etc) evaluated at the horizon. The only real deficiency in the tetrad we have chosen (and this is a matter of taste, not a matter of physics) is that while it is easy to write, and easy to interpret, and easy to calculate with, the 4-velocity $V$ is not geodesic (except in the rather special case where $c(r, \tilde{t}) \rightarrow c$ is a constant). Now in that special case not only is $V$ geodesic, but also $f=0$, the net flux seen by a freely falling observer located at the evolving horizon is zero. This leads us to ask whether this behaviour can be emulated by a slightly different choice of tetrad?

\section{General timelike-spacelike tetrad}

Consider now the 4-velocity

$$
\tilde{V}^{a}=\frac{1}{c \sqrt{1-\beta^{2}}}[1,-(v+c \beta), 0,0],
$$

where $|\beta(r, t)|<1$. This corresponds to an observer who is infalling with 3-velocity

$$
v_{3}(r, t) \equiv v(r, t)+c(r, t) \beta(r, t) .
$$

It is easy to check that $\tilde{V}$ is a unit timelike vector and easy to construct an appropriate spacelike vector that is orthonormal to it:

$$
\tilde{S}^{a}=\frac{1}{c \sqrt{1-\beta^{2}}}[-\beta, c+v \beta, 0,0] .
$$

This now allows us to construct a generic non-null tetrad (one element of the tetrad, $\tilde{V}$, is guaranteed to be timelike, the other three spacelike)

$$
e_{\hat{a}}^{a}=\left(\tilde{V}^{a}, \tilde{S}^{a}, \hat{\theta}^{a}, \hat{\phi}^{a}\right) .
$$

In terms of this tetrad, one now has

$$
\begin{aligned}
\tilde{\rho} & =\frac{G_{a b} \tilde{V}^{a} \tilde{V}^{b}}{8 \pi} \\
& =\frac{1}{4 \pi r c\left(1-\beta^{2}\right)}\left[c\left(1-\beta^{2}\right) \frac{m^{\prime}}{r}+\left(\beta+\sqrt{\frac{2 m}{r}}\right)^{2} c^{\prime}+\beta^{2} \partial_{t}\left(\sqrt{\frac{2 m}{r}}\right)\right] ;
\end{aligned}
$$




$$
\begin{aligned}
\tilde{f} & =\frac{G_{a b} \tilde{V}^{a} \tilde{S}^{b}}{8 \pi} \\
& =\frac{1}{4 \pi r c\left(1-\beta^{2}\right)}\left[-\beta \partial_{t}\left(\sqrt{\frac{2 m}{r}}\right)-\left\{\beta+\sqrt{\frac{2 m}{r}}\right\}\left\{1+\beta \sqrt{\frac{2 m}{r}}\right\} c^{\prime}\right] \\
\tilde{p}_{r} & =\frac{G_{a b} \tilde{S}^{a} \tilde{S}^{b}}{8 \pi} \\
& =\frac{1}{4 \pi r c\left(1-\beta^{2}\right)}\left[-c\left(1-\beta^{2}\right) \frac{m^{\prime}}{r}+\left(1+\beta \sqrt{\frac{2 m}{r}}\right)^{2} c^{\prime}+\partial_{t}\left(\sqrt{\frac{2 m}{r}}\right)\right] .
\end{aligned}
$$

Then at the evolving horizon, where $2 m=r$, we have

$$
\begin{aligned}
& \tilde{\rho}_{H}=\frac{1}{4 \pi r c\left(1-\beta^{2}\right)}\left[c\left(1-\beta^{2}\right) \frac{m^{\prime}}{r}+(1+\beta)^{2} c^{\prime}+\beta^{2} \frac{\dot{m}}{r}\right]_{H} ; \\
& \tilde{f}_{H}=\frac{1}{4 \pi r c\left(1-\beta^{2}\right)}\left[-\beta \frac{\dot{m}}{r}-(1+\beta)^{2} c^{\prime}\right]_{H} \\
& \tilde{p}_{r}^{H}=\frac{1}{4 \pi r c\left(1-\beta^{2}\right)}\left[-c\left(1-\beta^{2}\right) \frac{m^{\prime}}{r}+(1+\beta)^{2} c^{\prime}+\frac{\dot{m}}{r}\right]_{H} .
\end{aligned}
$$

The special case $\beta \rightarrow 0$ reproduces the results based on our 'natural' tetrad. From these results, we can see that the notion of 'flux across the horizon' is both subtle and less useful than one might at first suppose-the flux depends explicitly on the tetrad (effectively, one's choice of privileged observer), and indeed the 'flux across the horizon' can in many situations be made to vanish by choosing a suitable observer:

$$
\frac{\beta_{H}}{\left(1+\beta_{H}\right)^{2}}=-\left[\frac{r c^{\prime}}{\dot{m}}\right]_{H} \text {. }
$$

But even though the notion of flux is somewhat more subtle than expected, the evolution of the horizon as encoded in quantities such as $\left.\dot{m}\right|_{H}$ and $\mathrm{d} r_{H} / \mathrm{d} t$ is perfectly well defined and easy to deal with.

\section{Formalism for generic horizons}

We now show how to write the change in the area of the horizon as a function of the expansions defined above. First, choose a coordinate basis $(\theta, \phi)$ for the surface of the horizon at a given instant in time (on a given slicing). Then, the area of this 2-surface will be

$$
\mathcal{A}_{H}=\int \sqrt{\operatorname{det} h} \mathrm{~d} \theta \mathrm{d} \phi,
$$

where $h$ is the induced metric on the 2-sphere given by its embedding in the full 4D spacetime. For a spherically symmetric 2 -sphere this is

$$
\mathrm{d} s^{2}=r^{2}\left(\mathrm{~d} \theta^{2}+\sin ^{2} \theta \mathrm{d} \phi^{2}\right),
$$

and thus $\sqrt{\operatorname{det} h}=r^{2} \sin \theta$ giving the familiar $4 \pi r^{2}$ area. Now if we ask how this area varies as we move from one slice of the foliation to another we are led to consider

$$
\frac{\mathrm{D} \mathcal{A}_{H}}{\mathrm{~d} \lambda}=\frac{\mathrm{d} x^{a}}{\mathrm{~d} \lambda} \nabla_{a} \mathcal{A}_{H}=t^{a} \nabla_{a} \mathcal{A}_{H}=\mathcal{L}_{t} \mathcal{A}_{H},
$$


where $\lambda$ is a parameter labelling the foliations, and $t^{a}$ is chosen to be tangential to the horizon, but normal to the foliation and always future-pointing. To relate this expression to the expansions of the ingoing and outgoing null rays rewrite the above as

$$
4 \pi \mathcal{L}_{t} r_{H}^{2}=\frac{4 \pi}{\sin \theta} \mathcal{L}_{t} \sqrt{\operatorname{det} h}=2 \pi r_{H}^{2} h^{i j} \mathcal{L}_{t} h_{i j}
$$

Now identifying the intrinsic metric with the projection tensor $q_{a b}$ and writing $q_{a b}=$ $\hat{\theta}_{a} \hat{\theta}_{b}+\hat{\phi}_{a} \hat{\phi}_{b}$ where $\hat{\theta}_{a}$ and $\hat{\phi}_{a}$ are two orthonormal directions tangent to the 2 -surface, we get

$$
\frac{\mathrm{D} \mathcal{A}_{H}}{\mathrm{~d} \lambda}=4 \pi r_{H}^{2} q^{a b} \nabla_{a} t_{b}
$$

Here, $t^{a}$ can always be written as a linear combination of $\ell^{a}$ and $n^{a}$

$$
t^{a}=B \ell^{a}+C n^{a},
$$

where $B$ and $C$ are the coefficients and depend in general on $\lambda$. However on a given slice, for $t^{a}$ to be future-pointing, there are only five cases to consider:

\begin{tabular}{lcccc}
\hline$t^{a}$ outside $\ell^{a}$ & $t^{a} \propto \ell^{a}$ & $t^{a}$ between $\ell^{a}$ and $n^{a}$ & $t^{a} \propto n^{a}$ & $t^{a}$ inside $n^{a}$ \\
\hline$B>0$ & $B>0$ & $B>0$ & $B=0$ & $B<0$ \\
$C<0$ & $C=0$ & $C>0$ & $C>0$ & $C>0$ \\
\hline
\end{tabular}

Additionally, on the horizon,

$$
\frac{\mathrm{D} \mathcal{A}_{H}}{\mathrm{~d} \lambda}=4 \pi r_{H}^{2} C \theta_{n}
$$

Therefore, since $\theta_{n}$ is negative at the horizon by definition, the area $\mathcal{A}_{H}$ will be increasing in the first case, constant in the second case and decreasing in the other three cases.

\section{Discussion}

We have demonstrated a simple framework capable of handling black holes with rapidly evolving horizons. The only physical simplifying assumption was one of spherical symmetry, and the two mathematical simplifying assumptions were the adoption of a spherically symmetric slicing of spacetime and the use of a specific nonsingular coordinate chart (PainlevéGullstrand coordinates). The framework does not assume asymptotic flatness or stationarity, and we argue that these concepts are not required to define a useful concept of horizon. While exact spherical symmetry may not be an appropriate approximation to describe astrophysical black holes, we feel that this framework is useful in clarifying the basic concepts. In particular, once someone has understood the simple nature of the evolving horizon in Painlevé-Gullstrand coordinates, it is difficult to then become confused in other coordinate systems- the 'frozen' nature of the horizon in Schwarzschild coordinates is then very clearly seen to be a simple coordinate artefact due to that coordinate system becoming degenerate on the horizon.

Furthermore, this framework is useful in that any investigation of the black hole evaporation process must ultimately consider the essentially dynamic nature of the problem. While a particular choice of coordinates was used to facilitate some of the calculations, the simplicity of the results should in no way be seen as an exclusive property of the choice of coordinates. We have made no attempt to address the issue of singularity formation although Penrose's proof will remain valid within its assumptions. In this respect, it is important to 
note that the current work assumes a classical spacetime everywhere, and one would expect it to arise as some sort of limiting approximation to the models of Ashtekar and Bojowald who consider the explicit breakdown of the spacetime manifold description. To avoid a singularity (as we certainly have trapped surfaces), we must either violate the NEC (or have closed timelike curves or not have a manifold). Conservative approaches to avoiding singularities focus on the energy conditions and their violations. More radical approaches (either within loop quantum gravity or string models) effectively dispense with the spacetime manifold at sufficiently short distances. Still, before adopting the more radical approaches it is useful to see how far the standard manifold picture can be pushed.

One clear message that should be extracted form the current paper is that event horizons (at least in their precise mathematical definition as absolute horizons) are not essential for doing interesting black hole physics. Event horizons are mathematical abstractions that in many ways encode and presuppose too many technical assumptions - assumptions which may or may not have anything to do with reality. For physical black holes, evolving either due to accretion or Hawking evaporation, other notions of horizon seem to be more appropriate. Apparent horizons are sometimes useful but are also known to possess several technical deficiencies. Isolated horizons are in many ways 'too close' to being true event horizons (since no matter is allowed to cross the isolated horizon it cannot either grow or shrink). Hayward's trapping horizons, Ashtekar's dynamical horizons and the evolving horizons of this paper all capture key aspects of back hole physics without presupposing the existence of an event horizon. We would argue that the key feature of the evolving horizons of this paper is their relative simplicity and clarity, enabling one to build up a simple physical picture and a clear intuition.

\section{Acknowledgments}

This research was supported by the Marsden Fund administered by the Royal Society of New Zealand. We wish to thank the referees for useful comments and suggestions.

\section{Appendix A. Coordinate basis}

For completeness, here are the coordinate basis components of the Einstein tensor:

$$
\begin{aligned}
G_{t t} & =\frac{2 m^{\prime} c^{2}}{r^{2}}\left(1-\frac{2 m}{r}\right)+\frac{2 \dot{m} c}{r^{2}} \sqrt{2 m / r} \\
G_{t r} & =\frac{2 \dot{m}}{r^{2}}-\frac{2 m^{\prime} c}{r^{2}} \sqrt{\frac{2 m}{r}} \\
G_{r r} & =-\frac{2 m^{\prime}}{r^{2}}+\frac{2 c^{\prime}}{r c}+\frac{2 \dot{m}}{r^{2} c} \frac{1}{\sqrt{2 m / r}} \\
G_{\theta \theta} & =G_{\hat{\theta} \hat{\theta}} r^{2} \\
G_{\phi \phi} & =G_{\hat{\theta} \hat{\theta}} r^{2} \sin ^{2} \theta
\end{aligned}
$$

At the evolving horizon

$$
\begin{aligned}
G_{t t} & \rightarrow \frac{2 \dot{m} c}{r^{2}} ; \\
G_{t r} & \rightarrow \frac{2\left(\dot{m}-m^{\prime} c\right)}{r^{2}}
\end{aligned}
$$




$$
G_{r r} \rightarrow \frac{2 c^{\prime}}{r c}+\frac{2\left(\dot{m}-m^{\prime} c\right)}{r^{2} c}
$$

and

$$
\ell^{a} \rightarrow \frac{(1,0,0,0)}{c}
$$

This then easily yields (on the evolving horizon)

$$
G_{a b} \ell^{a} \ell^{b}=\frac{2 \dot{m}}{r^{2} c}
$$

which is a simple consistency check on the entire formalism.

\section{Appendix B. Null decomposition}

Let the indices $i, j, \ldots$ only take on the values 0,1 (so they lie in the $t-r$ plane). Then, spherical symmetry is enough to imply that

$$
T_{i j}=T_{0} g_{i j}+T_{+} \ell_{i} \ell_{j}+T_{-} n_{i} n_{j}
$$

Then, in terms of our natural tetrad

$$
T_{0}=\frac{-\rho+p_{r}}{2} ; \quad T_{+}=\frac{\rho+p_{r}-2 f}{4} ; \quad T_{-}=\frac{\rho+p_{r}+2 f}{4} .
$$

In terms of the Einstein tensor

$$
\begin{aligned}
& G_{0}=\frac{2 m^{\prime}}{r}-\frac{\dot{m}}{r c^{2}} \frac{1}{\sqrt{2 m / r}}-\frac{c^{\prime}}{r c}\left(1-\frac{2 m}{r}\right) ; \\
& G_{+}=\frac{\dot{m}}{2 r^{2} c} \frac{1}{\sqrt{2 m / r}}+\frac{c^{\prime}}{2 r c}\left(1+\sqrt{\frac{2 m}{r}}\right)^{2} ; \\
& G_{-}=+\frac{\dot{m}}{2 r^{2} c} \frac{1}{\sqrt{2 m / r}}+\frac{c^{\prime}}{2 r c}\left(1-\sqrt{\frac{2 m}{r}}\right)^{2} .
\end{aligned}
$$

At the apparent horizon

$$
\begin{aligned}
& G_{0} \rightarrow \frac{2 m^{\prime}}{r}-\frac{\dot{m}}{r c^{2}} \\
& G_{+} \rightarrow \frac{\dot{m}}{2 r^{2} c}+\frac{c^{\prime}}{r c} \\
& G_{-} \rightarrow \frac{\dot{m}}{2 r^{2} c}
\end{aligned}
$$

If we now go to a general radially infalling tetrad $v_{3}=v+\beta c$, then $G_{0}$ is independent of $\beta$ whereas the null vectors $\ell$ and $n$ are Doppler shifted

$$
\ell \rightarrow \tilde{\ell}=\ell \sqrt{\frac{1-\beta}{1+\beta}} ; \quad n \rightarrow \tilde{n}=n \sqrt{\frac{1+\beta}{1-\beta}} ;
$$

so that

$$
G_{+} \rightarrow G_{+} \frac{1+\beta}{1-\beta} ; \quad G_{-} \rightarrow G_{-} \frac{1-\beta}{1+\beta}
$$


In particular, the product $G_{+} G_{-}$is invariant under choice of radially infalling tetrad. This again demonstrates that while many statements that one might wish to make concerning the evolving horizon are tetrad dependent, there are also a number of useful quantities that are tetrad independent. In particular, note that if the evolving horizon is momentarily static, then since $\dot{m}=0$ we have $G_{-}=0$, and the stress-energy on the horizon exhibits a type of 'enhanced symmetry' [31] in that it takes on the form

$$
T_{i j}=T_{0} g_{i j}+T_{+} \ell_{i} \ell_{j}
$$

Under the significantly stronger assumption that the evolving horizon is static, at least for some finite time interval, and has been static long enough for a bifurcation 2-surface to form, then since $\ell \rightarrow 0$ on the bifurcation 2-surface, we have the much stronger result that

$$
T_{i j} \rightarrow T_{0} g_{i j}
$$

on the bifurcation 2-surface. This makes it clear that the 'enhanced symmetries' of [31] are very much related to the existence of both a finite-time translation symmetry and a bifurcation 2-surface. The enhanced symmetry (B.12) is already reduced as one moves away from the bifurcation 2-surface, and even the reduced symmetry (B.11) is at best only approximate once the horizon starts evolving.

\section{Appendix C. Special case: $c(r, t)=1$}

There is a special case of the formalism in which technical computations simplify even further-that is if we set $c(r, t)=1$. This is not a situation motivated by any particular physical principle: although it is true that $c(r, t)=1$ for both the Schwarzschild and ReissnerNordström solutions, there does not appear to be any deep reason for this. (The analogous property also holds for the Doran form of the Kerr solution [32], though again there seems to be no deep physical reason underlying this.) Nevertheless, the tremendous simplifications attendant on setting $c(r, t)=1$ are so significant, and do not seem to undermine the main points we are making regarding the non-perturbative evolution of horizons, that a brief summary may be warranted. (See the analogous discussion by Hayward in [14].)

Suppose we consider the metric

$$
\mathrm{d} s^{2}=-\left[1-v(r, \tilde{t})^{2}\right] \mathrm{d} \tilde{t}^{2}+2 v(r, \tilde{t}) \mathrm{d} r \mathrm{~d} \tilde{t}+\mathrm{d} r^{2}+r^{2} \mathrm{~d} \Omega^{2},
$$

and set $v(r)=\sqrt{2 m(r, \tilde{t}) / r}$. Then, the discussion of the evolving horizon is largely unaltered though now we have the simplification

$$
\kappa_{\ell}=\kappa_{n}=-v^{\prime}(r, t)=\frac{m / r^{2}-m^{\prime}}{\sqrt{2 m / r}} .
$$

In fact, we now have

$$
G_{a b} \ell^{a} \ell^{b}=G_{a b} n^{a} n^{b}
$$

which is enough to tell us without detailed computation that

$$
G_{a b} V^{a} S^{b}=0 \text {. }
$$

Indeed the 'comoving' tetrad defined by $V^{a}$ and $S^{a}$ is now geodesic, and the stress-energy tensor in this tetrad basis simplifies to

$$
\begin{aligned}
& \rho=\frac{G_{\hat{t} \hat{t}}}{8 \pi}=\frac{m^{\prime}}{4 \pi r^{2}} ; \\
& f=\frac{G_{\hat{t} \hat{r}}}{8 \pi}=0 ;
\end{aligned}
$$




$$
p_{r}=\frac{G_{\hat{r} \hat{r}}}{8 \pi}=-\frac{m^{\prime}}{4 \pi r^{2}}+\frac{\dot{m}}{4 \pi r^{2}} \frac{1}{\sqrt{2 m / r}} .
$$

The only 'complicated' component of the Einstein tensor is the transverse one:

$p_{t}=\frac{G_{\hat{\theta} \hat{\theta}}}{8 \pi}=\frac{G_{\hat{\phi} \hat{\phi}}}{8 \pi}=-\frac{m^{\prime \prime}}{8 \pi r}+\sqrt{\frac{2 m}{r}}\left[\frac{\dot{m}}{32 \pi m^{2} r}\left[m-r m^{\prime}\right]+\frac{1}{16 m \pi} \dot{m}^{\prime}\right]$.

At the apparent horizon, the only real simplifications are

$$
p_{r, H}=-\left[\frac{m^{\prime}-\dot{m}}{4 \pi r^{2}}\right]_{H},
$$

and

$$
p_{t, H}=\left[\frac{-m m^{\prime \prime}+m \dot{m}^{\prime}+\dot{m}\left(1-2 m^{\prime}\right) / 4}{4 \pi r^{2}}\right]_{H} .
$$

This particular class of spacetime geometries is slightly easier to deal with than those considered in the bulk of the article, and so is perhaps of some mathematical and pedagogical interest. However, it should be noted that the more fundamental issues of interest in this paper are the way in which we have explicitly shown how a suitable choice of coordinates completely side steps the 'frozen' nature of coordinate time at the Schwarzschild horizon and allows one to non-perturbatively formulate questions about the production and decay of evolving horizons.

\section{Appendix D. Vaidya spacetime}

The standard expression of the accreting Vaidya spacetime $[5,6]$ in advanced null coordinates is

$$
\mathrm{d} s^{2}=-\left(1-\frac{2 m(v)}{r}\right) \mathrm{d} v^{2}+2 \mathrm{~d} v \mathrm{~d} r+r^{2} \mathrm{~d} \Omega^{2}
$$

This spacetime represents collapsing null radiation, and the function $m(v)$ is an arbitrary nondecreasing function of its argument ${ }^{11}$. Note that the mass function is a function only of the advanced null coordinate $v .{ }^{12}$ We can transform to Painlevé-Gullstrand coordinates $\left(g_{r r}=1\right)$ by writing the null coordinate $v$ as a function of Painlevé-Gullstrand coordinates $\tilde{t}$ and $r$ : that is, write $v=v(\tilde{t}, r)$, whence

$$
\mathrm{d} v=\frac{\partial v}{\partial \tilde{t}} \mathrm{~d} \tilde{t}+\frac{\partial v}{\partial r} \mathrm{~d} r
$$

11 There is a related time-reversed 'shining star' solution for outgoing null radiation, which can be written as

$$
\mathrm{d} s^{2}=-\left(1-\frac{2 m(u)}{r}\right) \mathrm{d} u^{2}-2 \mathrm{~d} u \mathrm{~d} r+r^{2} \mathrm{~d} \Omega^{2},
$$

where $u$ is a retarded null coordinate and $m(u)$ is a non-increasing function of its argument. This can also be used as a model for a rather specific class of evaporating black hole. The comments below can immediately be carried over to this case by suitable changes in notation.

12 Unfortunately, the null coordinate $v$ occurring here has nothing to do with the function $v(r, \tilde{t})$ previously used to discuss the Painlevé-Gullstrand form of the metric. The usage and notation is unfortunately standard, and care must be taken to keep the two concepts distinct. 
Substituting, we obtain

$$
\begin{aligned}
\mathrm{d} s^{2}=-(1- & \left.\frac{2 m(v(\tilde{t}, r))}{r}\right)\left(\frac{\partial v}{\partial \tilde{t}}\right)^{2} \mathrm{~d} \tilde{t}^{2} \\
+ & {\left[-2\left(1-\frac{2 m(v(\tilde{t}, r))}{r}\right)\left(\frac{\partial v}{\partial \tilde{t}}\right)\left(\frac{\partial v}{\partial r}\right)+2\left(\frac{\partial v}{\partial \tilde{t}}\right)\right] \mathrm{d} \tilde{t} \mathrm{~d} r } \\
+ & {\left[-\left(1-\frac{2 m(v(\tilde{t}, r))}{r}\right)\left(\frac{\partial v}{\partial r}\right)^{2}+2\left(\frac{\partial v}{\partial r}\right)\right] \mathrm{d} r^{2}+r^{2} \mathrm{~d} \Omega^{2} }
\end{aligned}
$$

To obtain Painlevé-Gullstrand coordinates we demand $g_{r r}=1$ so

$$
\left[-\left(1-\frac{2 m(v(\tilde{t}, r))}{r}\right)\left(\frac{\partial v}{\partial r}\right)^{2}+2\left(\frac{\partial v}{\partial r}\right)\right]=1 .
$$

Solving this quadratic yields a first-order differential equation relating the coordinates $(v, r)$ and $(\tilde{t}, r)$ :

$$
\frac{\partial v}{\partial r}=\frac{1 \pm \sqrt{\frac{2 m(v(\tilde{t}, r))}{r}}}{1-\frac{2 m(v(\tilde{t}, r))}{r}}=\frac{1}{1 \mp \sqrt{\frac{2 m(v(\tilde{t}, r))}{r}}}
$$

Since $v$ is by construction regular at the evolving horizon we must take $\pm \rightarrow-$ and $\mp \rightarrow+$ above. Putting this back into equation (D.3) yields the Painlevé-Gullstrand form of the Vaidya solution

$\mathrm{d} s^{2}=-\left(1-\frac{2 m(v(\tilde{t}, r))}{r}\right)\left(\frac{\partial v}{\partial \tilde{t}}\right)^{2} \mathrm{~d} \tilde{t}^{2}+2 \sqrt{\frac{2 m(v(\tilde{t}, r))}{r}}\left(\frac{\partial v}{\partial \tilde{t}}\right) \mathrm{d} \tilde{t} \mathrm{~d} r+\mathrm{d} r^{2}+r^{2} \mathrm{~d} \Omega^{2}$.

In the original null coordinates, the evolving horizon occurs at

$$
r_{H}(v)=2 m(v)
$$

and there is clearly only a single horizon for any value of the null coordinate $v$. In terms of the Painlevé-Gullstrand coordinates $(\tilde{t}, r)$, the location of the horizon is given implicitly by

$$
r_{H}(v(\tilde{t}, r))=2 m(v(\tilde{t}, r)),
$$

where $v(\tilde{t}, r)$ is constrained by the differential equation (D.5). If the horizon is spacelike this condition can in principle have many solutions for fixed $\tilde{t}$, as the horizon can in principle move back and forth in Painlevé-Gullstrand time. Consider in particular the quantity $\partial m(v(\tilde{t}, r)) / \partial r$ :

$$
\frac{\partial m}{\partial r}=\frac{\mathrm{d} m}{\mathrm{~d} v} \frac{\partial v}{\partial r}=\frac{\mathrm{d} m}{\mathrm{~d} v} \frac{1}{1+\sqrt{\frac{2 m(t, r)}{r}}} .
$$

Then as $2 m \rightarrow r$ this becomes

$$
\frac{\partial m}{\partial r} \rightarrow m_{H}^{\prime}=\frac{1}{2} \frac{\mathrm{d} m}{\mathrm{~d} v}
$$

In order to violate equation (34) we would need $m_{H}^{\prime}>1 / 2$. But since in the Vaidya solution $\mathrm{d} m / \mathrm{d} v$ can be any non-negative function, this condition can certainly be violated. In short, for a spacelike evolving horizon 'outermost' in the sense of the $(v, r)$ null coordinates may not always coincide with 'outermost' in the sense of the $(\tilde{t}, r)$ Painlevé-Gullstrand coordinates. We emphasize that this is not an inconsistency in the formalism, merely one of the interesting coordinate artefacts one has to keep in mind. 


\section{References}

[1] Thorne K S, Price R H and Macdonald D A 1986 Black Holes: The Membrane Paradigm (New Haven: Yale University Press)

[2] Hawking S W 1974 Black hole explosions Nature 24830

Hawking S W 1975 Particle creation by black holes Commun. Math. Phys. 43199

Hawking S W 1976 Particle creation by black holes Commun. Math. Phys. 46206 (erratum)

[3] Birrell N D and Davies P C W 1982 Quantum Fields in Curved Space (Cambridge: Cambridge University Press)

[4] Oppenheimer J R and Snyder H 1939 On continued gravitational contraction Phys. Rev. 56455

[5] Vaidya P C 1953 The gravitational field of a radiating star Nature 171260 (republished as 1999 Gen. Rel. Grav. 31 121-35)

[6] Stephani H, Kramer D, MacCallum M, Hoenselaers C and Herlt E 2003 Exact Solutions of Einstein's Field Equations (Cambridge: Cambridge University Press)

[7] Ashtekar A and Galloway G J 2005 Some uniqueness results for dynamical horizons Adv. Theor. Math. Phys. 9 1-30 (Preprint gr-qc/0503109)

Ashtekar A and Krishnan B 2004 Isolated and dynamical horizons and their applications Living Rev. Rel. 710 (Preprint gr-qc/0407042)

Ashtekar A, Engle J, Pawlowski T and Van Den Broeck C 2004 Multipole moments of isolated horizons Class. Quantum Grav. 212549 (Preprint gr-qc/0401114)

Ashtekar A and Krishnan B 2003 Dynamical horizons and their properties Phys. Rev. D 68104030 (Preprint gr-qc/0308033)

Ashtekar A 2003 How black holes grow Preprint gr-qc/0306115

Ashtekar A, Corichi A and Sudarsky D 2003 Non-minimally coupled scalar fields and isolated horizons Class. Quantum Grav. 203413 (Preprint gr-qc/0305044)

Ashtekar A and Krishnan B 2002 Dynamical horizons: energy, angular momentum, fluxes and balance laws Phys. Rev. Lett. 89261101 (Preprint gr-qc/0207080)

Ashtekar A, Beetle C and Lewandowski J 2002 Geometry of generic isolated horizons Class. Quantum Grav. 191195 (Preprint gr-qc/0111067)

Ashtekar A, Beetle C and Lewandowski J 2001 Mechanics of rotating isolated horizons Phys. Rev. D 64044016 (Preprint gr-qc/0103026)

Ashtekar A, Beetle C, Dreyer O, Fairhurst S, Krishnan B, Lewandowski J and Wisniewski J 2000 Isolated horizons and their applications Phys. Rev. Lett. 853564 (Preprint gr-qc/0006006)

Ashtekar A, Fairhurst S and Krishnan B 2000 Isolated horizons: Hamiltonian evolution and the first law Phys. Rev. D 62104025 (Preprint gr-qc/0005083)

Ashtekar A and Corichi A 2000 Laws governing isolated horizons: inclusion of dilaton couplings Class. Quantum Grav. 171317 (Preprint gr-qc/9910068)

Ashtekar A, Beetle C and Fairhurst S 2000 Mechanics of isolated horizons Class. Quantum Grav. 17253 (Preprint gr-qc/9907068)

Ashtekar A, Beetle C and Fairhurst S 1999 Isolated horizons: a generalization of black hole mechanics Class. Quantum Grav. 16 L1 (Preprint gr-qc/9812065)

[8] Hayward S A 2004 Energy and entropy conservation for dynamical black holes Phys. Rev. D 70104027 (Preprint gr-qc/0408008)

Hayward S A 2004 Energy conservation for dynamical black holes Phys. Rev. Lett. 93251101 (Preprint gr-qc/0404077)

Hayward S A 2000 Black holes: new horizons Preprint gr-qc/0008071 (Published in 2000 The Ninth Marcel Grossman Meeting on Recent Developments in Theoretical and Experimental General Relativity, Gravitation and Relativistic Field Theories (Rome, 2000): Part A pp 568-80)

Mukohyama S and Hayward S A 2000 Quasi-local first law of black-hole dynamics Class. Quantum Grav. 172153 (Preprint gr-qc/9905085)

Hayward S A, Mukohyama S and Ashworth M C 1999 Dynamic black-hole entropy Phys. Lett. A 256347 (Preprint gr-qc/9810006)

Hayward S A 1998 Inequalities relating area, energy, surface gravity and charge of black holes Phys. Rev. Lett. 814557 (Preprint gr-qc/9807003)

Hayward S A 1998 Unified first law of black-hole dynamics and relativistic thermodynamics Class. Quantum Grav. 153147 (Preprint gr-qc/9710089)

Hayward S A 1996 Gravitational energy in spherical symmetry Phys. Rev. D 531938 (Preprint gr-qc/9408002)

Hayward S A 1994 Spin coefficient form of the new laws of black hole dynamics Class. Quantum Grav. 113025 (Preprint gr-qc/9406033) 
Hayward S A 1994 General laws of black hole dynamics Phys. Rev. D 496467

Hayward S A 1993 Marginal surfaces and apparent horizons Preprint gr-qc/9303006

[9] Hawking S W and Ellis G F R 1975 The Large Scale Structure of Space-Time (Cambridge: Cambridge University Press)

[10] Booth I 2005 Black hole boundaries Can.J. Phys. 831073 (Preprint gr-qc/0508107)

[11] Hawking S W 2004 "The way the information gets out seems to be that a true event horizon never forms, just an apparent horizon" Abstract of talk at GR17 (Dublin, Ireland, July 2004)

For a technical discussion see Hawking S W 2005 Information loss in black holes Phys. Rev. D 72084013 (Preprint hep-th/0507171)

[12] Ashtekar A and Bojowald M 2005 Black hole evaporation: a paradigm Class. Quantum Grav. 223349 (Preprint gr-qc/0504029)

[13] Hayward S A 2005 The disinformation problem for black holes (Conference version) Preprint gr-qc/0504037 Hayward S A 2005 The disinformation problem for black holes (Pop version) Preprint gr-qc/0504038

[14] Hayward S A 2006 Formation and evaporation of regular black holes Phys. Rev. Lett. 96031103 (Preprint gr-qc/0506126)

[15] Husain V and Winkler O 2005 Quantum resolution of black hole singularities Class. Quantum Grav. 22 L127 (Preprint gr-qc/0410125)

Husain V and Winkler O 2004 Quantum black holes Preprint gr-qc/0412039

Husain V and Winkler O 2005 Flat slice Hamiltonian formalism for dynamical black holes Phys. Rev. D 71104001 (Preprint gr-qc/0503031)

[16] Visser M 1995 Lorentzian Wormholes: From Einstein to Hawking (New York: AIP)

[17] Visser M 1992 Dirty black holes: thermodynamics and horizon structure Phys. Rev. D 462445 (Preprint hep-th/9203057)

[18] Wald R M and Iyer V 1991 Trapped surfaces in the Schwarzschild geometry and cosmic censorship Phys. Rev. D 44 R3719-22

[19] Schnetter E and Krishnan B 2006 Non-symmetric trapped surfaces in the Schwarzschild and Vaidya spacetimes Phys. Rev. D 73021502 (Preprint gr-qc/0511017)

[20] Roman T A and Bergmann P G 1983 Stellar collapse without singularities? Phys. Rev. D 28 1265-1277

[21] Courant R 1962 Partial Differential Equations (Methods of Mathematical Physics vol 2) ed R Courant and D Hilbert (New York: Wiley)

[22] Hernandez W C and Misner C W 1965 Observer time as a coordinate in relativistic spherical hydrodynamics Astrophys. J. 143 452-64

[23] Visser M 1993 Dirty black holes: entropy versus area Phys. Rev. D 48583 (Preprint hep-th/9303029)

[24] Visser M 1993 Dirty black holes: entropy as a surface term Phys. Rev. D 485697 (Preprint hep-th/9307194)

[25] Booth I, Brits L, Gonzalez J A and Van Den Broeck C 2006 Marginally trapped tubes and dynamical horizons Class. Quantum Grav. 23413 (Preprint gr-qc/0506119)

[26] Hochberg D and Visser M 1998 Dynamic wormholes, anti-trapped surfaces, and energy conditions Phys. Rev. D 58044021 (Preprint gr-qc/9802046)

Hochberg D and Visser M 1998 The null energy condition in dynamic wormholes Phys. Rev. Lett. 81746 (Preprint gr-qc/9802048)

Hochberg D and Visser M 1999 General dynamic wormholes and violation of the null energy condition Preprint gr-qc/9901020

[27] Roman T A 1986 Quantum stress energy tensors and the weak energy condition Phys. Rev. D 333526

[28] Roman T A 1988 On the 'averaged weak energy condition' and Penrose's singularity theorem Phys. Rev. D 37546

[29] Visser M 2003 Essential and inessential features of Hawking radiation Int. J. Mod. Phys. D 12649 (Preprint hep-th/0106111)

[30] Visser M 1998 Acoustic black holes: horizons, ergospheres and Hawking radiation Class. Quantum Grav. 151767 (Preprint gr-qc/9712010)

Barcelo C, Liberati S and Visser M 2005 Analogue gravity Living Rev. Rel. 812 (Preprint gr-qc/0505065)

[31] Medved A J M, Martin D and Visser M 2004 Dirty black holes: spacetime geometry and near-horizon symmetries Class. Quantum Grav. 213111 (Preprint gr-qc/0402069)

Medved A J M, Martin D and Visser M 2004 Dirty black holes: symmetries at stationary non-static horizons Phys. Rev. D 70024009 (Preprint gr-qc/0403026)

[32] Doran C 2000 A new form of the Kerr solution Phys. Rev. D 61067503 (Preprint gr-qc/9910099) Hamilton A J S and Lisle J P 2004 The river model of black holes Preprint gr-qc/0411060 